aelia rubescens Lindl. (Figura 1ALC) es una orquídea epífita o rupícola, que se distribuye desde México hasta Panamá. Crece principalmente en bosques tropicales secos, deciduos, y en ambientes de sabana (Halbinger y Soto, 1997; Trapnell et al., 2004; Trapnell y Hamrick, 2006), aunque es capaz de adaptarse a climas más templados (Halbinger y Soto, 1997). Son plantas con pseudobulbos casi redondos, comprimidos, con surcos irregulares y que generalmente presentan una hoja solitaria, terminal, rígida, coriácea, oblongo-elíptica, de hasta $21 \mathrm{~cm}$ de largo y $4 \mathrm{~cm}$ de ancho. Las espigas son terminales, de 20-60 cm de largo, con un racimo compacto de 3 a 15 flores que se abren en sucesión. Las flores son resupinadas, de color blanco, ligeramente rosado o rosado intenso, con una notable mancha violeta oscuro en la garganta del labelo. El tamaño de las flores varía entre 3 y $7 \mathrm{~cm}$ de diámetro, y cada flor individual tiene una duración aproximada de dos días; sin embargo, una espiga puede durar hasta

\section{Proliferación natural de LaElia RUBESCENS (ORCHIDACEAE) EN UN ÁREA suburbana de Pinar del Río, Cuba}

\author{
Alfredo García-González ${ }^{1}$ y Frander B. Riverón-Giró \\ El Colegio de la Frontera Sur (ECOSUR), Unidad Tapachula, \\ Tapachula, Chiapas, México. \\ 1Autor para correspondencia: alfredmx22@gmail.com
}

dos semanas. En su área de distribución, L. rubescens florece de agosto a noviembre (Halbinger y Soto, 1997).

En Cuba es una especie exótica ampliamente cultivada como ornamental en patios y jardines (Dietrich, 2007; Morales, 2010; Pérez et al., 2010a, b), y aunque es abundante en la Península de Yucatán, México (Halbinger y Soto, 1997), un territorio relativamente cercano al extremo occidental de Cuba, en la isla nunca ha sido reportada como especie nativa (Acuña, 1938; Mújica et al., 2000; Llamacho y Larramendi,
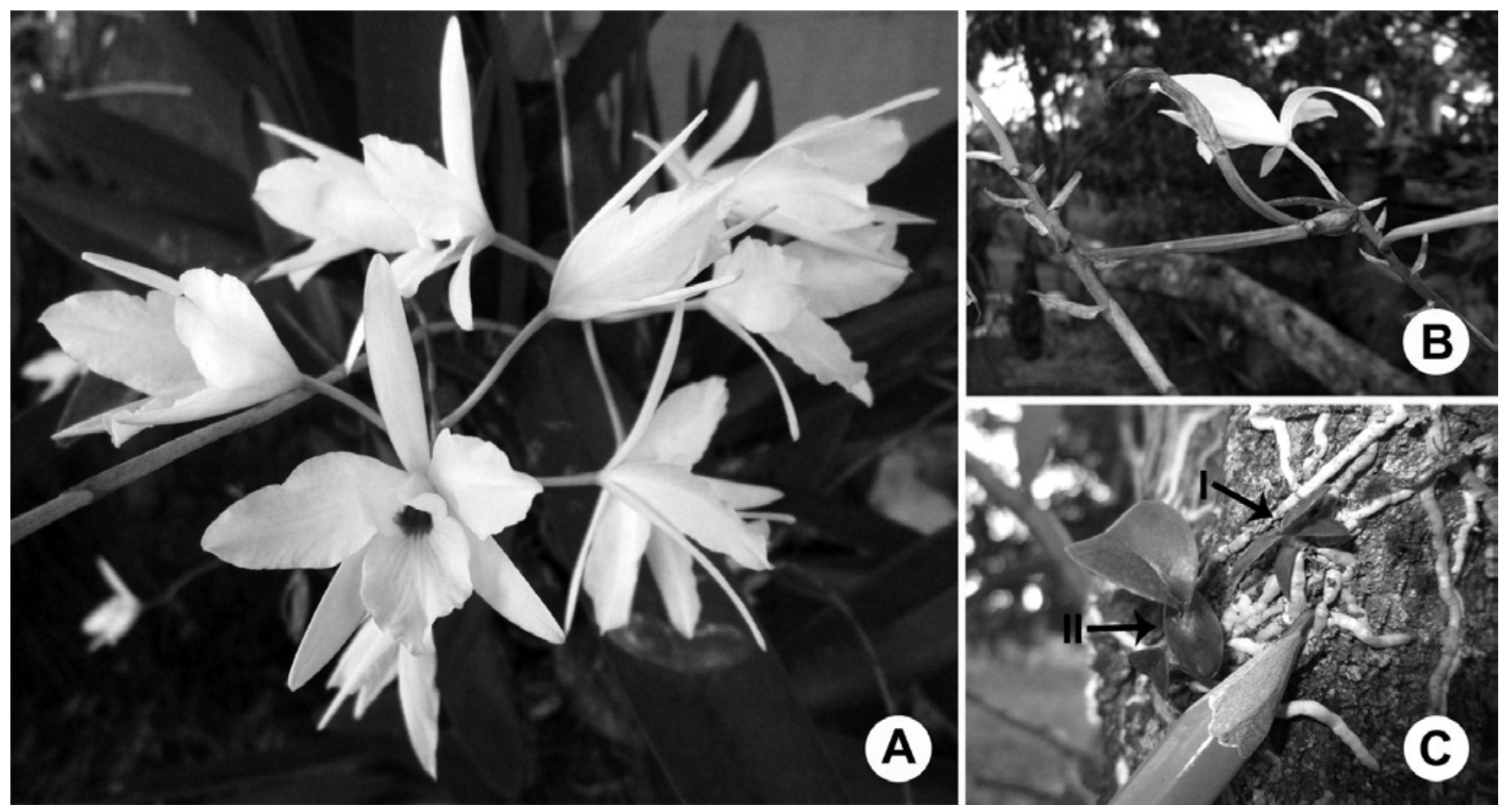

Figura 1. Laelia rubescens en la finca "La Juanita", Pinar del Río, Cuba. A) individuo adulto en floración, B) espigas con frutos inmaduros, C) individuos juveniles de menor tamaño (I) y juveniles de mayor tamaño (II) en árbol de mango (Mangifera indica). 
2005; Acevedo-Rodríguez y Strong, 2012; Ackerman, 2014). No obstante, probablemente ha logrado naturalizarse en algunas localidades del territorio nacional, por lo que se le considera una especie potencialmente invasora (González-Torres et al., 2012); aunque no existen datos, ni en el país se ha documentado un caso concreto de este fenómeno para Laelia rubescens. Esta situación es preocupante al tener en cuenta la vulnerabilidad de las floras insulares a las invasiones biológicas (Hughes et al., 1991; Lambdon et al., 2008), y es de vital importancia su monitoreo, para evaluar tempranamente los posibles impactos que puede causar esta especie en los ecosistemas naturales de Cuba y tomar medidas que contribuyan a mitigarlos.

En el Neotrópico se han documentado varios casos de orquídeas con comportamiento invasivo. Oeceoclades maculata (Lindl.) Lindl. es una especie terrestre de origen africano, que en México posiblemente es capaz de desplazar a Pelexia longipetiolata (Rchb. f.) Schltr. (Hágsater et al., 2005), mientras que en Puerto Rico probablemente está compitiendo con Prescottia stachyodes (Sw.) Lindl. y Wullschlaegelia calcarata Benth. (Cohen y Ackerman, 2009). En Cuba fue registrada en 1988, actualmente es una de las especies de orquídeas más comunes, con presencia en todas las formaciones vegetales del país (Llamacho y Larramendi, 2005), y está incluida en la lista de las 100 especies de plantas invasoras más nocivas (González-Torres et al., 2012). Generalmente ocupa tanto hábitats alterados como conservados, pero es más abundante y se expande con mayor facilidad en sitios alterados (García-González y Pérez, 2011). Spathoglottis plicata Blume también es una especie terrestre nativa de Asia tropical y del norte de Australia (Cribb y Tang, 1982), que se ha naturalizado en diferentes regiones del mundo (Bécquer y González-Torres, 2010). En Cuba está considerada como especie invasora, igualmente se incluye en la lista de las 100 especies más nocivas
(González-Torres et al., 2012), y ha sido reportada como naturalizada para el occidente (Mújica et al., 2000) y el oriente del país. Sin embargo, no ha sido evaluado su impacto en los ecosistemas naturales donde está presente (Bécquer y González-Torres, 2010). Oncidium poikilostalix (Kraenzl.) M.W.Chase \& N.H.Williams es una orquídea epífita nativa de Guatemala y Costa Rica (Behar y Tinschert, 1998), que exhibe comportamiento de maleza en plantaciones de café en México y puede estar desplazando a especies nativas (García-González et al., 2013).

En este trabajo se describe un caso concreto de proliferación natural de Laelia rubescens en un área suburbana de Pinar del Río, Cuba. Se da a conocer el número de individuos de la población, estructurados en etapas de crecimiento, los sustratos sobre los que crecen, las demás especies de epífitas vasculares (autóctonas y exóticas) con las que comparten el área, su posible polinizador y probable comportamiento de la especie en el futuro.

La proliferación natural de Laelia rubescens se produjo en un área sembrada principalmente de mango (Mangifera indica L.), naranja ( $\mathrm{C} i$ trus sinensis (L.) Osbeck) y otros frutales, en la finca "La Juanita" (22 28' 599" N, 083 37' 678'" O; 13 ha), situada $10 \mathrm{~km}$ al noreste de la ciudad de Pinar del Río, Cuba. El promedio anual de precipitaciones en el área es de $1,600 \mathrm{~mm}$ y las temperaturas promedio oscilan entre 23 y $25^{\circ} \mathrm{C}$ (Díaz y Cádiz, 2008), lo que coincide con las condiciones de hábitat que prefiere la especie en su zona de origen (Halbinger y Soto, 1997; Trapnell et al., 2004; Trapnell y Hamrick, 2006). El huerto donde se encuentra L. rubescens está rodeado por una matriz de terrenos agrícolas, árboles aislados, cercas "vivas" perimetrales formadas por especies de árboles nativos, y fragmentos de bosque de galería, rodeando cañadas y un río que pasa por la finca.

En el huerto, los propietarios de la finca tienen una pequeña colección de orquídeas epífitas autóctonas y exóticas, entre las que se encuentran individuos de Laelia rubescens. Todas las orquídeas crecen directamente sobre los troncos y ramas de los árboles frutales o en secciones de tallo de "guano prieto" (Acoelorraphe wrightii (Griseb. \& H.Wendl.) H.Wendl. ex Becc.; Arecaceae), que a su vez están colgados de estos árboles. Las primeras plántulas y juveniles pequeños de $L$. rubescens se detectaron en 2012, creciendo en una sección de A. wrightii, ocupada por una planta de Cyrtopodium punctatum (L.) Lindley (Orchidaceae), aunque no fue posible identificarlas en ese momento por su reducido tamaño y debido a que en la colección hay varias especies de orquídeas, aunque no todas son capaces de formar frutos y semillas.

Además de L. rubescens, en la colección están presentes y fructifican periódicamente (aunque nunca se han observado semillas germinando): Encyclia fucata (Lindl.) Britton \& Millsp., E. phoenicea (Lindl.) Neumann, Prosthechea boothiana (Lindl.) W.E.Higgins, Trichocentrum undulatum (Sw.) Ackerman \& M.W.Chase y Myrmecophila tibicinis (Bateman) Rolfe. Están presentes, pero no fructifican: Aerides odorata Lour., Cattleya labiata Lindl., C. punctatum, Dendrobium pierardii Roxb. ex Hook., D. superbiens Rchb. f., Encyclia bocourtii Múj.Benítez \& Pupulin y Papilionanthe teres (Roxb.) Schltr. También en el área crecen y se reproducen naturalmente diferentes especies de epífitas vasculares como: Ionopsis utricularioides (Sw.) Lindl. (Orchidaceae), Tillandsia balbisiana Schult. \& Schult. f., T. fasciculata Sw., T. flexuosa Sw., T. recurvata (L.) L. y T. utriculata L. (Bromeliaceae).

En 2013, luego de que algunos juveniles alcanzaron mayor talla, aumentaron el tamaño de sus hojas y desarrollaron pseudobulbo, fue posible identificarlos como Laelia rubescens. Posteriormente, se revisó detalladamente el árbol de mango (Mangifera indica) donde se localizaron la mayoría de las plántulas y juveniles, y los 
arboles cercanos. También se realizaron recorridos exploratorios en un radio de $80 \mathrm{~m}$ alrededor de los individuos adultos, con el fin de localizar nuevos juveniles y/o plántulas. Se recorrió esta superficie teniendo en cuenta que, aunque las semillas de la mayoría de las orquídeas se dispersan por el aire y pueden viajar largas distancias (Arditti y Ghani, 2000; Hágsater et al., 2005), tienen más probabilidades de caer cerca de la planta madre (Machon et al., 2003; Trapnell et al., 2004; Jersáková y Malinová, 2007; Winkler et al., 2009; Chung et al., 2011).

En la finca "La Juanita", Laelia rubescens regularmente florece (Figura 1A) y forma frutos (Figura 1B) todos los años, entre septiembre y diciembre. En la incipiente población de la finca se localizaron 47 individuos. No obstante, existe la posibilidad de que no se haya encontrado algún juvenil y/o plántula a causa de su pequeño tamaño y a la dificultad para revisar los árboles detalladamente debido a su gran porte. La población se estructuró en cuatro estadios de vida: adultos reproductores (plantas que han florecido al menos una vez), juveniles de mayor tamaño (ya presentan pseudobulbo), juveniles de menor tamaño (más grandes que una plántula, pero aún no presentan pseudobulbo) y plántulas (individuos $<0.5 \mathrm{~mm}$ de alto). Son tres adultos reproductores (plantas originales de la colección, crecen en secciones de Acoelorraphe wrightii; $6.38 \%$ de la población; Figura 1A), cinco juveniles de mayor tamaño $(10.64 \%$ de la población; 2 en sección de $A$. wrightii ocupada por Cyrtopodium punctatum, 1 en sección de A. wrightii ocupada por Encyclia bocourtii y 2 en árbol de Mangifera indica; Figura 1C), 12 juveniles de menor tamaño $(25.53 \%$ de la población; 4 en soporte de $C$. punctatum, 2 en soporte de E. bocourtii y 6 en $M$. indica; Figura 1C), y 27 plántulas $(57.45 \%$ de la población; 11 en soporte de $C$. punctatum, 1 en soporte de E. bocourtii y 15 en $M$. indica). Todas las plantas de L. rubescens crecen en un rango de alturas entre $1.30 \mathrm{~m}$ y $2.70 \mathrm{~m}$, en un área de $15 \times 15 \mathrm{~m}$ $\left(225 \mathrm{~m}^{2}\right)$.

Anualmente, la formación de frutos indica que Laelia rubescens probablemente encuentra polinizadores adecuados en la zona, ya que ha sido reportado que esta especie, en Costa Rica, depende obligatoriamente de agentes polinizadores para su fecundación, principalmente colibríes (Trapnell et $a l .$, 2004). En la finca "La Juanita" posiblemente es polinizada por zunzunes (Chlorostilbon ricordii Gervais), que es el único integrante de la familia Trochilidae que se encuentra en el área, de las tres especies reportadas para Cuba (Garrido y Kirkconnell, 2000); no obstante, nunca se ha observado a esta especie visitando la orquídea.

La germinación de semillas de Laelia rubescens, tanto en secciones de Acoelorraphe wrightii como en Mangifera indica, demuestra que la orquídea ha logrado asociarse con hongos micorrícicos presentes en estos medios, debido a que esta simbiosis es imprescindible para la germinación de sus semillas y el desarrollo de las plántulas (Walter, 1991; Otero et al., 2004; Trapnell y Hamrick, 2006; Jersáková y Malinová, 2007; Mújica et al., 2010). Probablemente $L$. rubescens es una especie flexible en cuanto a sus preferencias por micorrizas, como ocurre con otras especies de orquídeas (Hadley, 1970; Otero et al., 2004), por lo que logró adaptarse a hongos presentes en la corteza de $M$. indica o en las secciones de $A$. wrightii, algunos de ellos posiblemente simbiontes de Cyrtopodium punctatum y Encyclia bocourtii, que son las orquídeas que están adheridas a estos soportes. Estas orquídeas son especies autóctonas cubanas y fueron recolectadas en la naturaleza.

Laelia rubescens es una especie exitosa en paisajes altamente modificados por intervención humana, llegando a ser abundante en pastizales abiertos con árboles aislados (Trapnell et al., 2004; Trapnell y Hamrick, 2006). Por ejemplo, en Costa Rica, en la hacienda "Solimar", un rancho ganadero caracterizado por árboles aislados y grupos pequeños de árboles distribuidos por pastizales abiertos, se muestrearon tres árboles aislados en los que se encontraron más de 370 plantas (Trapnell et al., 2004). Por lo antes expuesto, y teniendo en cuenta que en la finca "La Juanita" $L$. rubescens ha logrado franquear las principales barreras reproductivas de las orquídeas (polinizadores y hongos micorrícicos; Cohen y Ackerman, 2009), que cada cápsula de orquídea puede contener miles o incluso millones de semillas, que sus semillas son pequeñas, que tienen grandes espacios de aire internos que les permiten flotar en la columna de aire, facilitando su dispersión (Arditti y Ghani, 2000; Hágsater et al., 2005), y que las semillas de orquídeas también pueden ser transportadas en el plumaje de las aves (Arditti y Ghani, 2000); es factible que en el futuro sean colonizados otros árboles del huerto y probablemente árboles aislados, árboles de las cercas "vivas" perimetrales y árboles de los fragmentos de bosque de galería que se encuentran en la finca.

\section{Agradecimientos}

Agradecemos a la familia García, propietarios de la finca "La Juanita", por el apoyo brindado. A los revisores anónimos por su tiempo y sus recomendaciones. A la organización IDEA WILD por el equipamiento proporcionado, fundamental para la realización de este trabajo.

\section{Literatura citada}

Acevedo-Rodríguez P. y Strong M.T. 2012. Catalogue of seed plants of the West Indies. Smithsonian Contributions to Botany. Number 98. Smithsonian Institution Scholarly Press, Washington, D.C.

Acuña J. 1938. Catálogo descriptivo de las orquídeas cubanas. Boletín Técnico No. 60. Estación Experimental Agronómica Santiago de Las Vegas. Secretaría de Agricultura de Cuba, Provincia de La Habana. 
Ackerman J.D. 2014. Laelia. En: Ackerman J.D. Coor. Orchid flora of the Greater Antilles, pp. 224-226. Memoirs of the New York Botanical Garden, Volume 109. The New York Botanical Garden Press, Nueva York.

Arditti J. y Ghani A.K.A. 2000. Numerical and physical properties of orchid seeds and their biological implications. Tansley Review 110. New Phytologist 145:367-421.

Bécquer E.R. y González-Torres L.R. 2010. Spathoglottis plicata (Orchidaceae) invade los ecosistemas del norte de Cuba Oriental. Bissea 4:1.

Behar M. y Tinschert O. 1998. Guatemala y sus orquídeas. Bancafé, Grupo Financiero del País, Ciudad de Guatemala.

Chung M.Y., Nason J.D. y Chung M.G. 2011. Significant demographic and finescale genetic structure in expanding and senescing populations of the terrestrial orchid Cymbidium goeringii (Orchidaceae). American Journal of Botany 98:2027-2039.

Cohen I.M. y Ackerman J.D. 2009. Oeceoclades maculata, an alien tropical orchid in a Caribbean rainforest. Annals of Botany 104:557-563.

Cribb P.J. y Tang C.Z. 1982. Spathoglottis (Orchidaceae) in Australia and the Pacific Islands. Kew Bulletin 36:721-729.

Díaz L.M. y Cádiz A. 2008. Guía Taxonómica de los Anfibios de Cuba. Vol. 4. Abc Taxa, Bruselas.

Dietrich H. 2007. Orchidaceae I: Parte general. Flora de la República de Cuba 12:3-114.

García-González A., Damon A., Iturbide F.A. y Olalde-Portugal V. 2013. Reproduction of Oncidium poikilostalix (Orchidaceae), potentially invading coffee plantations in Soconusco, Chiapas, México. Plant Ecology and Evolution 146:36-44

García-González A. y Pérez R. 2011. La comunidad orquideológica en la Reserva de la Biosfera Sierra del Rosario, Cuba. Revista de Biología Tropical 59:1805-1812.

Garrido O.H. y Kirkconnell A. 2000. Field Guide to the Birds of Cuba. Cornell University Press, Nueva York.

González-Torres L.R., Rankin R. y Palmarola, A. 2012. Plantas invasoras en Cuba. Bissea 6:1-132 (número especial 1).

Hadley G. 1970. Non-specificity of symbiotic infection in orchid mycorrhiza. New Phytologist 69:1015-23.

Hágsater E., Soto M., Salazar G., Jiménez R., López M. y Dressler R. 2005. Las Orquídeas de México. Instituto Chinoin, A.C., México, D.F.

Halbinger F. y Soto M. 1997. Laelias of México. Orquídea (Méx.) 15:1-160.

Hughes F., Vitousek P.M. y Tunison T. 1991. Alien grass invasion and fire in the seasonal submontane zone of Hawai'i. Ecology 72:743-746.

Jersáková J. y Malinová T. 2007. Spatial aspects of seed dispersal and seedling recruitment in orchids. New Phytologist 176:237-241.

Lambdon P.W., Lloret F. y Hulme P.E. 2008. Do non-native species invasions lead to biotic homogenization at small scales? The similarity and functional diversity of habitats compared for alien and native components of Mediterranean floras. Diversity and Distributions 14:774-785.

Llamacho J.A. y Larramendi J.A. 2005. Las Orquídeas de Cuba. Greta Editores, Lleida.

Machon N., Bardin P., Mazer S.J., Moret J., Godelle B. y Austerlitz F. 2003. Relationship between genetic structure and seed and pollen dispersal in the endangered orchid Spiranthes spiralis. New Phytologist 157:677-687.

Morales A. 2010. Las orquídeas que se cultivan con mayor frecuencia en Cuba.
Revista del Jardín Botánico Nacional 30-31:159-167.

Mújica E., Raventós J. y González E. 2010. Análisis de la selección de sustrato por parte de Dendrophylax lindenii (Orchidaceae) en Cabo San Antonio, Península de Guanahacabibes, Pinar del Río, Cuba. Lankesteriana 9:533-540.

Mújica E., Pérez R., Bocourt J.L., López P.J. y Ramos T.M. 2000. Géneros de Orquídeas Cubanas. Felix Varela, La Habana.

Otero J.T., Ackerman J.D. y Bayman P. 2004. Differences in mycorrhizal preferences between two tropical orchids. Molecular Ecology 13:2393-2404.

Pérez L., Fuentes V.R. y González-Torres L. R. 2010a. Flórula ornamental del municipio Boyeros, ciudad de La Habana, Cuba. Revista del Jardín Botánico Nacional 30-31:169-186.

Pérez L., Fuentes V.R. y González-Torres L. R. 2010b. Condiciones de cultivo, técnicas de propagación y distribución de las especies cultivadas con fines ornamentales en el municipio Boyeros, ciudad de La Habana, Cuba. Revista del Jardín Botánico Nacional 30-31:187201.

Trapnell D.W. y Hamrick J.L. 2006. Variety of phorophyte species colonized by the neotropical epiphyte, Laelia rubescens (Orchidaceae). Selbyana 27:60-64.

Trapnell D.W., Hamrick J.L. y Nason J.D. 2004. Three-dimensional fine-scale genetic structure of the neotropical epiphytic orchid, Laelia rubescens. Molecular Ecology 13:1111-1118.

Walter D.S. 1991. Orchidaceae. En: Janzen D.H. Ed. Historia Natural de Costa Rica, pp. 286-295, Editorial de la Universidad de Costa Rica, San José.

Winkler M., Hülber K. y Hietz P. 2009. Population dynamics of epiphytic orchids in a metapopulation context. Annals of Botany 104:995-1004. 